\title{
INFORMES
}

\author{
RESUMEN DEL SEMINARIO SOBRE TEMAS \\ DE INDUSTRIALIZACION CELEBRADO EN EL COLEGIO \\ DE MEXICO EL JUEVES 15 DE NOVIEMBRE DE $1979^{1}$
}

El Sr. Víctor L. Urquidi dio la bienvenida a los asistentes e indicó que la reunión tenía por objeto - además de despedir al Ing. Tulio de Andrea, con motivo de separarse de Naciones Unidas y ausentarse de Méxicocambiar impresiones sobre tema de interés permanente para El Colegio, como es el de las perspectivas de industrialización de México puesto que forma parte de la estrategia de desarrollo económico; que no se pretendía que fuera un seminario formal ni ocasión para profundizar demasiado en corto tiempo, pero sí para un cambio de impresiones sobre los aspectos sobresalientes del tema; que con este fin el Ing. Martínez del Campo - quien estaba muy interesado en estas cuestiones pues eran parte esencial de su trabajo en El Colegio de México- y él mismo, habían preparado un esquema de dos puntos generales y otros que si había tiempo se entraría a considerar.

Agregó que sí había una meta de industrialización —que a largo plazo no se sabía cuál fuera, pero que a corto y mediano plazos estaba expresada en el Plan Nacional de Desarrollo Industrial de la Secretaría de Patrimonio y Fomento Industrial-, aparte de la congruencia de los fines con los medios, importaba saber cuáles eran los problemas que la experiencia indica que aún subsisten o que podrían crearse en mayor escala y llegar a obstaculizar el alcance de dichas metas, cualesquiera que fueren. Al respecto se deseaba aprovechar la amplia experiencia nacional e internacional de las personas allí reunidas.

El Ing. Tulio de Andrea agradeció la invitación del Presidente de El

1 Se sesionó de las 14:00 a las 19:00 horas con la asistencia de las siguientes personas: Ing. Tulio de Andrea, Lic. Federico Ballí, Ing. Oscar M. Becerril, Lic. Guillermo Becker, Dr. Gerard K. Boon, Lic. Luis Bravo Aguilera, Ing. Isaías Flit, Ing. Bruno Guevara, Dr. Danilo Jiménez, Lic. Adrián Lajous V., Lic. Benito Rey Romai, Ing. Luis Unikel, Sr. Víctor L. Urquili, Ing. Eduardo Wygard e Ing. Manuel Martínez del Campo. 
Colegio de México y a continuación se abrió el debate, en el que intervinieron activamente todos los participantes, con los siguientes resultados principales: ${ }^{2}$

1. Se considera muy dudoso que pueda alcanzarse la meta de $\operatorname{Lima}^{3}$ relativa a que, para el año 2000 , los países del Tercer Mundo elaboren en su conjunto el $25 \%$ de la producción industrial mundial; se estima que es más factible una cifra del orden del $13.5 \%$ ( en la actualidad producen alrededor de1 $9 \%$ ). Por otra parte, se pone en duda también el que alcanzar dicho $25 \%$ sea de un beneficio neto proporcional a dicha cifra para los países en desarrollo, debiendo considerarse sobre todo el aspecto cualitativo, esto es, qué se habrá de producir, por quién, cómo, etcétera. ${ }^{4}$

2. Respecto a las metas nacionales, el Plan de Desarrollo Industrial de la Secretaría de Patrimonio y Fomento Industrial (SEPAFIN), publicado en abril de 1979, ofrece indicaciones en cuanto a los plazos corto y mediano; sin embargo, conviene, tratar de ver un poco más allá, por lo menos hasta el año 2000. El Plan Nacional de Desarrollo Industrial ${ }^{5}$ se ha venido discutiendo públicamente desde marzo pasado; es de suponer que, conforme el tiempo avance, se puedan ir presentando algunos retrasos, porque en la implementación mucho se depende de los proyectos del sector privado. El Estado, por su parte, interviene también en forma directa en algunos renglones o sectores, como es el caso de la industria mediana y pequeña, pero aquí también se tiene la impresión de que los esfuerzos son insuficientes en relación a la magnitud de las necesidades (aunque no necesariamente en cuanto a las metas del Plan).

3. Algunos aspectos importantes a considerar a largo plazo en las metas del desarrollo industrial son, por ejemplo, la fuerte dinámica demográfica del país y las crecientes necesidades de absorción de fuerza de trabajo en empleo productivo; en esto hay que tener en cuenta la tendencia natural - conforme avanza el desarrollo general del país- a que la mano de obra se desplace de las actividades primarias a los sectores secundario y terciario.

4. El Plan es muy claro y preciso en cuanto a la meta principal, que consiste en acercar al país a resolver su problema básico de empleo ${ }^{6}$ hacia finales de este siglo, esto es, en una generación, y tratar de resolver aspectos del subempleo. Es ésta una meta impresionantemente ambiciosa,

2 Estos resultados no constituyen un consenso de opiniones ni son conclusiones del grupo, sino puntos de vista expresados durante el debate. Se presentan aproximadamente en el orden en que se hicieron y en general son individuales, pero en algunos casos se combinaron varias aportaciones cuando eran complementarias. En el texto se señalan los principales casos en que hubo divergencia.

3 Segunda Conferencia General de la Organización de las Naciones Unidas para el Desarrollo Industrial (onudi), Lima, Perú, 12-26 de marzo de 1975.

4 Dicho de otra manera, podría contribuir más al progreso y bienestar de los países subdesarrollados producir, por ejemplo, sólo el 15\% (para el año 2000) si esa producción fuera sobre todo de bienes de consumo popular y de capital, se realizara de manera preponderante por empresas netamente nacionales, por medio de tecnologías adecuadas y cuyo valor agregado se distribuyera equitativamente entre consumidores (vía precios), trabajadores (vía salarios, etc.) y empresarios (vía utilidades razonables).

${ }^{5}$ En estas notas se le denominará indistintamente Plan, Plan Industrial o Plan de Desarrollo Industrial.

6 Sobre este punto hubo opiniones divergentes. 
pero se apoya en el hecho de que estamos viviendo la discontinuidad histórica que plantea el encontrarse ahora con abundancia de recursos petroleros. La mayoría de los países petroleros ha tratado de partir de la disponibilidad de este recurso para desarrollar su sector industrial; México está en condiciones de invertir parcialmente esta secuencia, ya que tiene una base industrial relativamente avanzada, lo que le ofrece perspectivas que difícilmente podrían haberse previsto inclusive hace muy pocos años.

5. Uno de los aspectos más importantes del Plan Industrial es que por primera vez existe oficialmente y con una estructura formal. Sin embargo, debe entenderse que en países como México es muy difícil la planeación general y sectorial, ya que con frecuencia se presenta un conjunto de elementos y situaciones dadas a los que pudieran denominarse el "antiplan". Además, en el caso presente, si bien el Plan Industrial ha sido dotado de su propio marco general - derivado de las políticas de la actual administración - es indudable que hace falta un documento equivalente que defina explícitamente cuestiones como lo que se desea en materia de administración de recursos, de inversiones extranjeras, de relaciones internacionales de desarrollo tecnológico, de énfasis en la capitalización, de relaciones obrero-patronales y de posición salarios/precios.

Por otra parte, el Plan permite por primera vez hablar de metas que trascienden un sexenio. En este sentido habrá que vincular dicho Plan con la perspectiva de ingreso de México al GaTT, en cuanto a las concesiones de comercio que al respecto se acuerden en su oportunidad.

6. Las principales limitaciones del Plan son: primero, el débil vínculo entre la agricultura y la industria, entre otras cosas por la falta de un plan agropecuario o algo equivalente; segundo, la falta de articulación, ya a nivel técnico más preciso, del crecimiento industrial con el incremento de la ocupación no sólo en términos de volumen sino de calidad de empleo; y, en tercer lugar, la falta de eslabonamiento entre pequeña y mediana, por una parte, y gran industria, por otra. Se ha tendido más a la integración vertical que a la horizontal, y ello es un inconveniente porque limita el crecimiento de la pequeña y mediana industria que podría especializarse para un mercado seguro como es el de la gran industria. Posiblemente ello se deba al impuesto en cascada del $4 \%$ que limita esa integración horizontal, lo cual quizá se supere con el impuesto al valor agregado que entrará en vigor en enero de 1980 .

7. En el presente caso no debe tomarse la palabra "plan" literalmente, pues se habla de una estrategia y como tal es difícil empezar a medir sus resultados a menos de un año de planteada. En la mecánica misma del Plan está previsto que se revisarán anualmente las proyecciones, lo que en estos momentos se está llevando a cabo en la Secretaría de Patrimonio y Fomento Industrial. Una de las críticas que se han hecho al Plan es que el modelo macroeconómico que se utilizó para elaborar estas proyecciones no es muy explícito en cuanto al aspecto financiero, pero espera que ello quedará resuelto con la actual revisión y cuyos resultados habrán de conocerse en marzo de 1980.

8. Es más difícil pensar que sea la agricultura la que absorba mano de obra, aun cuando este sector creciera a tasas muy elevadas. En esto, la elección de subsectores es determinante tanto en la agricultura como en 
la industria; en efecto, si todo fuera petroquímica sería muy distinto en cuanto a creación de empleos que si todo fuera industria forestal, y otro tanto ocurre en la agricultura, pues hay cultivos que son más mecanizables que otros.

Por otra parte, el ritmo de crecimiento de la economía es muy impor. tante en cuanto al grado de solución de este tipo de problemas. Hay economistas que opinan que si se diera un crecimiento económico del $8 \%$ (lo que haría imperativo que la industria creciera al $12 \%$ aproximadamente) se eliminarían el desempleo y el subempleo.

Ahora, el objetivo no puede ser sólo éste, sino que además, por ejemplo, tienen que ser satisfechas las necesidades básicas de la población; si se tiene una gran planta industrial y no se puede producir a precios adecuados lo que la población necesita para sobrevivir a un nivel decoroso, aquélla no tiene razón de ser y un plan que no atendiera a éste y a otros objetivos fundamentales no podría calificarse de tal.

El propósito principal del desarrollo no está en el crecimiento de la economía, ni está simplemente en decidir si éste se obtiene por la vía de la industrialización o de la modernización agrícola, etc., sino que ese propósito fundamental de desarrollo es llegar a un nivel cada vez más elevado de bienestar general.

Desde hace unos treinta años se viene diciendo que un crecimiento económico y una mayor industrialización traería aparejada la elevación de los niveles de vida de las mayorías y esto casi no ha sucedido. Por lo tanto, ese modelo de desarrollo debe revisarse a fondo pues ha acrecentado la brecha entre la gran masa de la población y los grupos de estratos superiores donde se da la concentración de la riqueza y del bienestar.

9. Es insuficiente todo lo que se ha hecho en México a favor de la industria mediana y pequeña. Las órdenes de magnitud del apoyo - crediticio y de otra índole - han sido insignificantes; cuando se hablaba hace algunos años de que se habían concedido créditos a 3000 empresas, las necesidades eran por lo menos 50 o 100 veces mayores. ${ }^{7}$ Además, los mecanismos que se han diseñado en los últimos dos años para su fortalecimiento son sumamente complejos y existen demasiados organismos que no operan con la debida coordinación. Además de establecer el marco general de apoyo, como lo viene haciendo, el gobierno podría propiciar que las empresas paraestatales realizaran compras preferenciales a empresas medianas y pequeñas, como se ha hecho en la India, Estados Unidos y otros países.

10. Uno de los grandes problemas de la industria, no sólo en México sino en la mayoría de los países en desarrollo, es el bajo aprovechamiento de la capacidad instalada; en la industria mediana y pequeña ello sucede casi siempre por problemas de ingeniería industrial, como son el mantenimiento de equipo, la existencia de puntos de estrangulamiento y otras cuestiones similares. Son muy pocas las industrias que trabajan tres turnos, en lo cual incide todo el mecanismo de las leyes laborales, lo que con vistas a resolver o atenuar un problema tan importante debiera revisarse a fondo.

7 Recientemente, el FOGAIN ha citado que los créditos que concedió en un año ascendieron a unos 6000 millones de pesos, pero que la demanda real era de 18000 millones aproximadamente. 
11. Es muy difícil entender, para quien no esté familiarizado con la mecánica de la política mexicana, cómo es posible que se publique un Plan Industrial sin que exista otro de carácter global, ${ }^{8}$ o por lo menos una estrategia general de desarrollo debidamente explicitada. Además, se percibe una serie de fuerzas contrarias que, como se mencionó en el punto 5 , podría ser calificada de "antiplan" y que desemboca en la concentración del poder, con la adquisición de algunas industrias clave por consorcios internacionales, con el crecimiento del sector financiero en manos privadas, con la inadecuada distribución de las ganancias nacionales, así como otras resultantes que -utilizando el lenguaje en el sentido que se da en México a algunos términos - constituyen de hecho un proceso contrarrevolucionario.

12. Un elemento importante, que no encuentra expresión en el Plan, es el relativo a la normalización y en general al control de la calidad de los productos; esto ha sido muy bien comprendido en países que han tenido un avance industrial notable, como Japón y Brasil. En el primero de estos países los "círculos de calidad" han sido la base para que de una producción de "pacotilla" todavía en los años cincuenta se haya pasado en los años sesenta y setenta a tener una calidad industrial que compite con la mejor del mundo; además de dichos "círculos" hubo toda una estructura, con un depurado sistema de normalización y disposiciones del gobierno de ese país, como las restricciones a la exportación de artículos que no alcanzaran las normas de calidad. En Brasil, los empresarios de São Paulo ayudaron a crear y apoyaron un sistema sólido de normalización y racionalización tanto a nivel nacional como dentro de sus empresas.

13. El Plan Industrial, en ausencia del global, ha tratado de definir su propio marco; así, procura establecer reglas y parámetros por lo que se refiere al comportamiento de los salarios, de la actividad monetaria y fiscal, etc. Esto, en realidad, es uno de sus principales méritos, pues haber esperado a que se tuviera un plan global de desarrollo habría dado lugar a que se retrasara aún más el Plan Industrial.

Sin embargo, existen, algunas contradicciones; una de ellas es que si bien ataca el modelo anterior de "desarrollo estabilizador", sigue basándose en un esquema de acumulación de capital en que las clases pobres serán cada vez más pobres, pues se prevé que los salarios reales van a descender durante los tres primeros años del Plan. ${ }^{9}$

14. Se requiere cambiar la mentalidad del empresario privado en México, quien durante mucho tiempo ha estado acostumbrado a una protección total que no estaba sujeta a normas de calidad ni a márgenes razonables de ganancia, lo que le ha impedido concurrir a los mercados internacionales. Esa tarea de cambio de mentalidad empresarial puede llevar una o dos generaciones, por lo que es importante, como lo sugiere la temprana aparición del Plan Industrial —respecto del global- iniciar la puesta en marcha de proyectos aún antes de recibir los ingresos derivados del excedente petrolero.

8 En marzo de 1979, la Secretaría de Programación y Presupuesto anunció un Plan Global de Desarrollo, pero en realidad se dispuso sólo de la metodología. Fosteriormente se ha vuelto a mencionar, pero aún no se dispone de él.

9 Uno de los participantes indicó que esta apreciación se basaba en los documentos de apoyo al Plan. 
15. En México se ha discriminado siempre a favor de un uso intensivo del capital: existió la Ley de Industrias Nuevas y Necesarias, después la Regla 14, luego los decretos de descentralización de industrias y ahora el nuevo esquema de Certificados de Promoción Fiscal. Todas las políticas de estímulo a la inversión dan por resultado la sustitución de mano de obra por equipo, sobre todo ante los problemas laborales, debido en parte a lo avanzado de nuestra legislación en la materia; por todo ello, el desarrollo industrial no significa forzosamente un incremento en el empleo, lo cual implica también la necesidad y conveniencia de fomentar las industrias de exportación, pues de todos modos para hacer eficiente el sector ha de limitarse la incorporación de mano de obra al mismo.

16. En general, sigue faltando mano de obra calificada, prácticamente a todos los niveles, pero en particular en la del obrero especializado, lo cual es doblemente negativo para el país y para el desarrollo industrial porque, por una parte, muchos desempleados no encuentran ocupación remunerada por falta de esa capacitación $\mathrm{y}$, por otra, numerosos puestos de trabajo que reclaman cierta especialización están ocupados por personas sin los conocimientos o experiencia necesarios para hacerlos realmente productivos.

17. Se ha hablado de dar prioridad a la producción de artículos básicos, pero en la instrumentación del Plan se ha omitido establecer una política crediticia de tasas preferenciales, así como otras de estímulos fiscales y precios, que orienten los recursos de capital hacia estas actividades; al habilitar el programa de 17 productos básicos lo único significativo que se ha hecho, es abastecer la región políticamente más impactante, o sea el Distrito Federal, y con ello se sigue propiciando la concentración de la población en la zona metropolitana. Ésta es otra de las contradicciones que se presentan.

18. Es importante decidir si se desea tener grandes grupos industriales o no; si se desea que sigan proliferando los ALFA, ICA, etc., se tienen que establecer los marcos de estímulo con una continuidad adecuada al caso, pues no es posible cambiar de posición de tiempo en tiempo, ya que la formación de esos grupos requiere muchos años. Esto tendría que ver también con la definición que se haga respecto de las empresas transnacionales, pues si en alguna forma se frena su proliferación o expansión en México, hay que formar como contrapartida grupos nacionales.

19. Están razonablemente bien delimitadas las funciones del sector público y el privado, respectivamente, en cuanto al desarrollo industrial. Incluso los campos están precisados, como sucede con algunas ramas sobre todo de las industrias pesadas y química. Además, una situación de crecimiento acelerado como la que se prevé en los próximos años hace posible la concurrencia de todos los sectores, casi sin restricción, pues si la industria crece al $12 \%$ anual quiere decir que se triplicará en 10 años y aumentará diez veces para el año 2000 , respecto de su nivel actual.

20. Los problemas del desarrollo industrial son múltiples y muy complejos; algunos, más que problemas son parámetros o condiciones dadas y su clasificación puede atender a diversos criterios. En el esquema presentado a los participantes se optó por la clásica división entre niveles: empresarial y nacional; pero hay otros criterios como el de la continuidad o recurrencia, grado de interrelación, importancia relativa, etc. Un aspecto que preo- 
cupa es el hecho de que algunos de los problemas del desarrollo industrial del país parecen no tener solución - a pesar de las mútiples medidas a lo largo de muchos decenios-; es importante encontrar las causas y obstáculos, en relación con toda planeación y acción futura.

21. Al analizar el sector industrial y considerar sus perspectivas de desarrollo, debe tenerse presente que en nuestro sistema económico-social existen elementos - figuras, formas y vicios- que datan de la época prehispánica y que no podrán desarraigarse en corto tiempo; cuestiones como la tenencia de la tierra, la política laboral, el proteccionismo ante la presión de la competencia del exterior, la posición frente al capital extranjero, etc., que han conformado nuestra manera de ser y de pensar y lo cual no podrá cambiar sino gradualmente. $Y$ es dentro de este esquema, dentro de esas condiciones de la política nacional, que habrá que enmarcar el desarrollo -económico e industrial- de México.

Algunos ejemplos concretos de lo anterior son la pesca y los bosques; para citar sólo dos. Respecto de la primera, treinta y tantos años de forma y estructura de las relaciones, de reserva de especies a determinados grupos y sectores, con muchos problemas de capacidad, tecnología, organización, financiamiento, indiscutiblemente que condicionan al sector. En cuanto a los bosques, se dispone de recursos importantes, sobre todo en coníferas, que no están explotados debidamente; pero la solución de ello, en la parte industrial, está condicionada a problemas de tenencia, estructura ejidal y otros, de solución política complicada.

22. La ley de Transferencia de Tecnología que se promulgó en el año de 1973 ha auxiliado mucho al sector industrial mexicano, puesto que le ha dado el poder de negociación de que antes carecía frente a los consorcios internacionales, que transfieren normalmente la tecnología; sin embargo, en México la legislación sobre tecnología no ha sido lo suficiente previsora como para proyectarse de modo que el Plan de Desarrollo Industrial tenga los resultados que se estiman.

Por ejemplo, una de las disposiciones, la que se refiere a secrecía, señala un límite máximo para preservarla, lo cual es suficiente en la mayoría de las ocasiones, pero no en todas. Otro caso está en la Ley de Patentes y Marcas, la que teóricamente no ha eliminado la protección - con su nueva figura del "certificado de invención" en lugar de las patentes tradicionales-, pero que en la práctica, debido a lo impreciso de ese nuevo concepto, da lugar a que tanto mexicanos como extranjeros se muestran poco dispuestos a revelar sus invenciones en nuestro país.

23. Un Plan Industrial necesita de un plan tecnológico y subsecuentemente de una política tecnológica y una estrategia de implementación; uno de los objetivos de estos planes debería ser el de reducir la dependencia tecnológica, lo cual sería posible, en cierta medida, por medio de la iniciación de un proceso de cambio tecnológico en el país.

El proceso de cambio tecnológico local puede adelantarse al desarrollar la industria de bienes de capital, la cual tiene que ser muy selectiva en un principio en cuanto al grado de refinamiento - que debe ser bajo-, de los productos por elaborar, así como respecto del mercado por atender, debiéndose dar preferencia al sector semiformal (empresas medianas y pequeñas) de la industria y otras actividades económicas. Cuando se atienda, en esta fase inicial, al sector formal (industria grande) es conveniente 
escoger los tipos más sencillos de bienes de capital e ir avanzando gradualmente en la elaboración de otros más refinados.

24. El Plan Nacional de Desarrollo Industrial es débil en cuanto a que sólo utiliza estímulos, y no hace uso de prohibiciones, en el problema de la distribución geográfica de la actividad económica y en particular en cuanto a la concentración excesiva en determinados puntos de la República, de manera notable en la ciudad de México. Sin embargo, debido a las implicaciones jurídicas y políticas tan importantes que pudieran presentarse, tal vez no sea posible que el Plan Industrial vaya más allá.

En este sentido se manifiestan algunas de sus limitaciones - al no estar vinculado con un plan global, por no existir éste- que dan por resultado también una falta de congruencia con otros planes sectoriales, como es el caso del Plan de Desarrollo Urbano. En efecto, este último plantea 11 zonas prioritarias, mientras que el Plan Industrial señala 16 zonas industriales prioritarias adicionales y deja abierta la posibilidad de negociar con los gobiernos de los estados otras áreas prioritarias denominadas "zonas 2", 10 que junto con las otras zonas prioritarias de diversos planes sectoriales como turismo, pesca, etc., hace que el número total de dichas zonas pueda ser tan elevado que implique una dilución tal de las inversiones que llegue a nulificar su efecto.

25. El empresario privado en general requiere rentabilidad inmediata ya que, de manera frecuente, se trata de grupos familiares que han aportado su patrimonio y necesitan de las utilidades de sus negocios para subsistir, o por lo menos para no percibir menores ingresos que los que obtenían por concepto de ventas de valores o bienes raíces. Por otro lado, el empresario mayor, con más ilustración y conocimiento de la actividad industrial, también requiere rentabilidad inmediata, no sólo cuando aporta su propio capital, sino cuando utiliza el crédito bancario, pues tiene que pagar los intereses a tasas relativamente elevadas y hacer frente a riesgos e incertidumbre. En el fondo toda esta situación de grandes y pequeños empresarios poco dispuestos a correr riesgos tiene que ver, en parte, con el raquitismo del mercado de valores; en efecto, si la mayor parte de las empresas se instalara o creciera a través de emisiones públicas, el riesgo se limitaría y no habría tanta urgencia por obtener rentabilidad inmediata.

Pero indudablemente que en el inversionista privado mexicano hay un deseo marcado de obtener prontos beneficios al grado de que por ejemplo, cuando el sector oficial inicia un proyecto e invita a grupos privados a participar, éstos se abstienen si dicho proyecto no tiene perspectivas de rendir utilidades antes de los primeros tres años.

Lo que un inversionista privado espera obtener comúnmente es algo asî como el doble de lo que obtendría en valores de renta fija. Ahora, que después de impuestos, los valores de renta fija pueden dar un $15 \%$, el inversionista en México espera por lo menos el 30 por ciento.

En cuanto al lapso que puede dejar transcurrir sin percibir utilidades, esto va en relación con el tamaño de la empresa; en una pequeña el propietario espera utilidades desde el primer año; en una mediana no esperaría hasta el segundo año, y en una empresa grande ese lapso de espera podría ser de tres o cuatro años, pero todo ello en función de la facilidad de acceso al crédito. Esto está ligado a la tradición existente en México y en toda América Latina, de una rotación rápida del capital y de una acelerada 
obtención de utilidades, lo cual se relaciona con situaciones de fragilidad política y económica, y de una mentalidad comercial que sólo podrá cambiar en una o dos generaciones.

26. Otro aspecto que distorsiona en forma sensible la estructuración del aparato productivo y que imposibilita la satisfacción de la demanda social, es el enorme gasto publicitario del segmento industrial, que se rige por la ley de la maximización de la utilidad y la acelerada recuperación de las inversiones.

Basta advertir la enorme publicidad, sobre todo en la televisión, toda ella encaminada a satisfacer una demanda suntuaria, o por lo menos una demanda de las clases media y alta, para darse cuenta del desvío que implica en la satisfacción de las necesidades sociales básicas. Obviamente es más rentable producir "corn flakes" que maíz, y bebidas alcohólicas que alimentos proteicos.

27. Se formula la interrogante de si las maquiladoras, como fenómeno relativamente nuevo en el mundo en materia de exportación de manufacturas, ofrecen un medio de industrializarse en países como México. Sin duda contribuyen positivamente a la generación de empleo y divisas, pero surgen dudas en cuanto a la magnitud de los inconvenientes que traen consigo, sobre todo en los aspectos sociales. Se estima que el sector público no ha tomado aún una posición bien definida sobre qué hacer con la industria de maquila, qué apoyos dar o qué desincentivos aplicar.

28. Surge la duda en cuanto a si la meta principal en materia tecnológica deba ser la de reducir la dependencia o la de avanzar lo más posible en la utilización eficiente de la misma, ya sea nacional o extranjera. En la mejor utilización quedaría incluido el proceso de adaptarla de acuerdo con las necesidades de producción y consumo nacionales, tales como las de capacidad instalada por planta, prototipos de productos $y$ requerimientos de amplios sectores de la población.

29. Uno de los aspectos centrales del acelerado proceso de industrialización en países como Corea del Sur ha sido la transferencia directa de tecnología - de empresa a empresa- combinada con un crecimiento hacia afuera.

Teniendo en cuenta el éxito observado en Corea, país pequeño casi sin recursos naturales, no puede menos que sentirse optimismo por el futuro desarrollo industrial de México, que para el año 2000 puede llegar a ser una potencia en la fabricación de manufacturas, dado que dispone de petróleo, de la cercanía de importantes mercados exteriores, de un amplio territorio y creciente población.

Otro de los aspectos positivos de la experiencia coreana que México tal vez podría tomar ${ }^{10}$ es que los grandes proyectos, ya sea que se ubiquen en el sector privado o en el gobierno, tienen o se les da carácter nacional con todo el apoyo consiguiente.

30. Para contrarrestar la salida extraordinaria de divisas con motivo del alza del precio del petróleo, a partir de 1973-1974 los coreanos empezaron a construir obras de ingeniería y plantas industriales en el Oriente Medio,

10 Sobre este punto, de la asimilación de experiencias coreanas en México, hubo distintas opiniones, pues algunos participantes apuntaron que habría que modificar la mentalidad del industrial mexicano y algunos factores conexos. 
habiendo en la actualidad unos 60 a 70000 coreanos en esa región, desde donde exportan alrededor de 3000 a 4000 millones de dólares adicionales.

Por otra parte, en Corea se están ampliando aún más algunas industrias, entre ellas la del automóvil ${ }^{11}$ del que se producen unas 100000 unidades anuales, con exportación del $90 \%$. El precio de exportación es de 2100 dólares, pero el de venta en el interior asciende a 7100 dólares, con un impuesto de placas y tenencia de 410 dólares al año, o sea que no se están imitando pautas de consumismo y todo se canaliza al ahorro.

31. Se sugirió que si el Plan Industrial señala que se devolverá cierto tipo de impuestos a quienes exporten tecnología, debería hacerse extensiva esta franquicia fiscal a quienes la produzcan para ser utilizada aquí en México, así como a aquellos que, importándola, la adecúen a las necesidades locales, pues no basta desescalar, ya que entonces los costos, que están en razón exponencial inversa con respecto al tamaño, se proyectan de manera desproporcionada. ${ }^{12}$

\section{PROGRAMA DE INVESTIGACIONES SOCIALES SOBRE POBLACIÓN EN AMÉRICA LATINA (PISPAL)}

El Programa de Investigaciones Sociales sobre Población en América Latina (PISPAL) tiene como objetivos fundamentales desarrollar teoría y metodología adecuadas a las características de la región; promover la realización de investigaciones que atiendan a las líneas prioritarias definidas; canalizar fondos de investigación que permitan ampliar la capacidad de investigación de los centros nacionales y la incorporación y adiestramiento de investigadores noveles; y facilitar el intercambio de información básica y la discusión de los resultados y conclusiones alçanzadas a través del análisis científico, organizado para ello reuniones de trabajo, seminarios y otras actividades.

Las líneas de estudio definidas como prioritarias para los fines del Programa son:

Primera linea. Estilos de desarrollo, diferenciación social y regional, estrategias de sobrevivencia y dinámica poblacional.

a) Los estilos de desarrollo y su relación con las estrategias de repro-

11 La marca es "Pony", carro pequeño de línea europea y tecnología japonesa.

$12 \mathrm{Se}$ comentó que en el Plan Industrial existan incentivos fiscales para aquellas empresas que adquieran maquinaria, y que otros organismos del Gobierno Federal asignen apoyos diversos al desarrollo de la tecnología nacional, su utilización y su venta en el exterior. 
ducción biológica en las unidades familiares de grupos social y regionalmente diferenciados y la estructura y crecimiento de la población.

b) Los estilos de desarrollo en su relación con estrategias de reproducción material en las unidades familiares de grupos social y regionalmente diferenciados, los flujos migratorios y la distribución espacial de la población.

c) Los estilos de desarrollo en su relación con las estrategias de supervivencia familiar de grupo social y regionalmente diferenciados y las condiciones de reproducción de la fuerza de trabajo urbana.

Segunda linea. Efectos sociales de la dinámica de la población, en particular condicionantes demográficos de la viabilidad de los estilos de desarrollo.

Tercera línea. Estilos de desarrollo, procesos político-ideológicos, cuestión poblacional y políticas con efectos demográficos.

El Programa opera a través de convocatorias periódicas en las que científicos sociales de la región presenta propuestas de investigación sobre temas considerados de interés directo para los fines de PISPAL. Los proyectos son evaluados por jurados externos al Programa. La decisión definitiva acerca de los proyectos a ser financiados está a cargo de un Sub-Comité ad-hoc compuesto por cinco miembros del Comité del Programa.

Los científicos sociales que concursen deberán pertenecer al cuerpo profesional de una institución de investigación social de la región, la cual deberá proporcionar al concursante apoyo de infraestructura y en general el respaldo de la institución.

Para mayores informes sobre PISPAL dirigirse a: PISPAL, El Colegio de México, Camino al Ajusco No. 20, Apartado Postal 20-671, México 20, D. F. 